\title{
Führungs- und \\ Verantwortungskultur auf Station Wo Menschen sich als Menschen begegnen: Eine Frage der Augenhöhe
}

Stationen sind die zentralen Leistungserbringer im direkten Patientenkontakt im Krankenhaus. Im geschützten Umfeld der Station soll der Patient Zutrauen fassen und sich für den Heilungsprozess öffnen. Und tatsächlich - wenn wir uns die Beschwerden und Lobs anschauen - Patientenzufriedenheit wird stark von den Erfahrungen der Patienten auf Station geprägt. Wie er sich im Funktionsbereich oder bei Einschleusung in den OP behandelt gefühlt hat, scheint dagegen von eher nachrangiger Bedeutung zu sein.

Wir hören immer wieder von 2 Arten von Patienten: Die einen, die sich den lieben langen Tag lang beschweren und die Arbeitszeit der Mitarbeitenden auf Station zumindest gefühlt enorm belasten und das andere Extrem: Patienten, die den offenbar immer gehetzten Mitarbeitenden nicht zur Last fallen wollen und ihre Bedürfnisse und Sorgen in sich hinein schweigen. Beide Extreme sind Alltagsbeispiele von wenig gemeinsamer Augenhöhe im Patientenkontakt.

Wie der Patient seine Zufriedenheit beurteilt, hängt stark von seiner Erwartung an die stationäre Versorgung ab. Erhofft er sich Sicherheit, dass er in seinen Ängsten Aufklärung erfährt und sieht aber allenfalls für ein paar Minuten am Tag für ihn ansprechbares Fachpersonal, trifft dies nicht seine Erwartung. Zufriedenheit entsteht, wenn der Patient von den Mitarbeitern positiv überrascht wird. Wenn er gar Vertrauen fasst und eine persönliche Beziehung mit einem Mitarbeiter aufbaut, dann ist die Weiterempfehlung der Klinik schon fast ein sicheres Ereignis.

Doch die Realität in den Kliniken sieht oft anders aus. Mitarbeiter finden selbst nicht die innere Zufriedenheit und Ruhe, den Patienten entsprechende Zuwendung geben zu können. Es lässt sich feststellen, dass dies auch der größte Unzufriedenheitsfaktor für das Personal selbst ist.

Dies kann nur geändert werden, wenn auf Station klare Verantwortungsbereiche mit klaren Führungsprofilen geschaffen werden und die Positionen mit engagierten Persönlichkeiten besetzt werden.

\section{Das Diktat der Effizienz und die} Hürde der grenzauflösenden Verantwortungskultur

Welche Einrichtung hat ihn in den letzten Jahren nicht mitgemacht? Einen massiven Stellenabbau insbesondere des Pflegepersonals. Gleichzeitig haben sich durch Demografie und die medizin-technischen Möglichkeiten, aber auch durch zunehmendes Gesundheitsbewusstsein, die Patientenzahlen deutlich erhöht. In der Bettenauslastung wurden die individuellen Verweildauerreduktionen mehr als kompensiert. Ergebnis ist eine massive Leistungsverdichtung in der stationären Krankenversorgung. Unkoordinierte Entlassungen, neu aufgenommene Patienten, die auf den Fluren auf ihre Betten warten, Hektik unter den Mitarbeitern ein unstrukturierter Stationsablauf kann Folgen mit sich bringen, welche die Mitarbeiter unangemessen belasten und die Patientenversorgung beeinträchtigen.

Die Arbeitsbelastung auf Station wird durch Fluktuation bzw. hohen Krankheitsausfall weiter verschärft. Zeit für kurze Gespräche mit den Patienten oder 15-Minuten-Pausen werden unter diesem gewachsenen Zeit- und Leistungsdruck als nicht mehr machbar empfunden. So äußern Mitarbeiter, es sei teils nicht mehr möglich, die Werte des Krankenhauses - der Patient im Mittelpunkt! - zu leben. Zu sehr sind Ärzte und Pflegende damit beschäftigt, die anfallenden Arbeiten auf Station in der geforderten Zeit bzw. in ihrer Schicht zu bewältigen. Neben den originären Kerntätigkeiten müssen umfangreich dokumentarische oder fachfremde Tätigkeiten übernommen werden. 
Der Wandel hin zu geordneten Management-Strukturen, die Entlastung für alle Beteiligten mit sich bringen, bedarf einer gesunden Balance zwischen den Bedürfnissen von Patienten und Mitarbeitern. Zudem müssen sich die neuen Strukturen an der Effektivität der Stationsabläufe orientieren. Eine Lösung hierfür besteht darin, die Abläufe der verschiedenen Berufsgruppen und Organisationsbereiche an den Schnittstellen rund um die Patientenversorgung auf der Station eng miteinander zu verzahnen. Das Potenzial, unnötige Arbeitsschritte zu reduzieren und Aufgaben sinnvoller zu verteilen, ist dabei immer wieder erstaunlich hoch. Die Herausforderung liegt dabei darin, die berufsgruppenübergreifenden Prozesse in die Alltagsroutine zu überführen. Dies ist nur möglich, wenn tatsächlich ein Wandel hin zu einer neuen Verantwortungskultur stattfindet, in der sich die Mitarbeiter aller Berufs- und Hierarchiegruppen auf Station auf Augenhöhe begegnen. Nur so werden die vereinbarten Absprachen und Prozesse tatsächlich gelebt und auf den Patienten hin ausgerichtet.

Stationen sind geprägt von einer hohen Anzahl an Schnittstellen, Abteilungsgrenzen sowie Grenzen zwischen Berufsgruppen, die in sich hierarchisch strukturiert sind. Die Einführung erfolgreicher Stationsstrukturen erfordert daher eine Vernetzung von Verantwortungsbereichen und eine offene, prozessbezogene Aufgabenverteilung. Für die Einhaltung berufsgruppenübergreifender Managementstrukturen zu sorgen und Mitarbeitende individuell $\mathrm{zu}$ führen und damit intrinsische Motivation zu fördern - die Aufgabenstellung ist für die Führungskraft auf Station gewaltig. Weil die Aufgabe für die Funktionsfähigkeit des Gesamtsystems aber so wichtig ist, kommt der Auswahl der richtigen Führungskräfte eine tiefgreifende Bedeutung zu.

\section{Stationen auf Augenhöhe managen} Die Überwindung der seit Jahrzehnten existierenden Berufsgruppengrenzen und die einhergehende Zusammenführung der wesentlichen Expertisen im Stationskontext können gelingen, indem Bewusstheit in die gemeinsame und koordinierte Führungsverantwortung der Station durch Arzt und Pflege gelegt wird. Hierzu eignet sich das Konstrukt von Managementteams, die aus dem stationsverantwortlichen Oberarzt und der
Stationsleitung bestehen. Problemstellungen, die innerhalb der Station auftauchen, können im Managementteam auf Augenhöhe gemeinsam berufsgruppenübergreifend reflektiert und gelöst werden. Dass es hierbei nicht zuletzt um sehr viel Geld geht, zeigt die Betrachtung der summierten DRG-Pauschalen der Patienten einer Station. Nimmt man hier den Anteil, den das InEK für die Normalstation kalkuliert, so ergeben sich für die meisten 30-40-Bettenstationen Jahresumsätze in der Größendimension von 2-3 Millionen Euro. Das heißt, Stationen können mit kleinen bis mittelständischen Unternehmen verglichen werden. Welches Unternehmen würde jedoch seine Führung unkoordiniert einer wechselnden Zusammensetzung von Fachexperten überlassen? Spätestens an dem Vergleich wird plastisch vor Augen geführt, wie wichtig es ist, dass ein Rahmen für professionelles Management auf Ebene der Stationen geschaffen wird.

\section{Mitarbeiterbindung: Meist sind es immaterielle Leistungsanreize}

Funktionierende Führungsstrukturen sind nicht nur unter dem Gesichtspunkt der klaren Verantwortlichkeiten und effektiver Hand-in-Hand-Arbeit wichtig, sondern auch im Hinblick auf die Personalgewinnung und Personalbindung. Es wird zunehmend schwieriger, Fachkräfte im Unternehmen zu halten und neue zu gewinnen. Dabei entscheiden weder Geld noch Status in erster Linie darüber, ob ein Mitarbeiter in seinem Job zufrieden ist. Es sind meist immaterielle Leistungsanreize, die Mitarbeiter an das Unternehmen binden. Führungskräfte müssen die Bedürfnisse und Motive der Mitarbeiter ernst nehmen und offen ihre Bereitschaft bekunden, diese in ihrem Fortkommen zu unterstützen. In einem Mitarbeitergespräch sollte somit nicht nur über den Erfolg diskutiert werden, sondern auch über zukünftige Ziele. Wenn die Interessen des Mitarbeiters regelmäßig in die Diskussion einbezogen werden, fühlt sich der Mitarbeiter wertgeschätzt und als wichtigen Teil eines großen Ganzen [1]. Führungskräfte, welche ihre Mitarbeiter durch Teamgeist und Visionen anleiten und fördern, werden von den Mitarbeitern dadurch belohnt, dass diese Verantwortung übernehmen. Für die sorgfältige und wirksame Gestaltung der Beziehungen in einem Verantwortungssystem ist ein kontinuierlicher Dialog zum Thema Verantwor- tung, Ziele und Ergebnisse notwendig. Das kostet zunächst einmal Zeit, die bewusst investiert werden muss. Doch nur so lässt sich auf Dauer Zeit sparen. Verantwortung wird nicht immer selbstverständlich von allen Mitarbeitern übernommen, auch wenn dies der kommunizierte Wille der Führungskraft ist. Dazu braucht es fachlich und persönlich die Fähigkeit und den Willen, das übertragene Anliegen zum eigenen Ziel zu machen und umzusetzen [4]. Insbesondere im mittleren Management der Kliniken werden hierfür die Grundlagen gelegt. Die Veränderung von Führungsstrukturen bricht tradierte Rollenverständnisse auf. Dies geht in der Regel mit Ängsten oder inneren Widerständen einher. Flankierende Schulungen oder Einzelcoachings, welche die Führungskräfte auf ihre neue Rolle vorbereiten, sind daher zielführend und unterstützen den Veränderungsprozess. Sie werden ihre Wirksamkeit vor allem dann entfalten, wenn die oberen Führungskräfte vom Ergebnis überzeugt sind und - in Worten und Taten - das gemeinsame Miteinander vorleben. Engagement von Seiten der Führungskraft wird für das Team wahrnehmbar und mit Motivation und Einsatz belohnt [7].

\section{Die mittlere Führungsebene: Emanzipation auf Augenhöhe}

Eine gute mittlere Führungsebene braucht eine starke, unterstützende Führungsebene, um Entscheidungen schnell herbeizuführen. Zwischen der mittleren und der oberen Führungsebene sind die vertrauensvolle Zusammenarbeit und gute Kommunikation der jeweiligen Zielvorstellungen bedeutend. Im entsprechenden Engagement von Pflegedirektion und Chefarzt/ Klinikdirektion beantwortet sich die Gretchenfrage - hier unterscheiden sich Lippenbekenntnisse von tatsächlichen Verantwortungssystemen. Als Spiegel für den Organisationserfolg lässt sich wiederum die Zufriedenheit der Mitarbeiter in der täglichen Arbeit sehen - die am Ende immer ein Abgleich gegen die eigenen Erwartungen der Mitarbeitenden darstellt und dementsprechend im Dialog ernst zu nehmen ist. Dieser mittleren Führungsebene wurde in der Vergangenheit zu wenig Bedeutung zugesprochen. Dabei kommt gerade ihr eine der wichtigsten Rollen im Krankenhaus zu - wenn nicht vielleicht sogar die wichtigste. Welchen Wert das Managementteam in der Organisation einnimmt, wird deutlich, wenn die Arbeit der Geschäftsführung be- 
trachtet wird. Hier werden zum Teil strategische Entscheidungen getroffen, ohne davor die „internen Experten“ zu Rate zu ziehen. Diese Entscheidungen sind oftmals im Stationsalltag nur schwer umsetzbar. Die mittlere Führungsebene ist die einzige, die eine Brücke zwischen der Unternehmensleitung und den Anforderungen des Tagesbetriebes schlagen kann. Sie ist nahe genug an der Geschäftsführung, um Einfluss auf strategische Entscheidungen zu nehmen als auch nahe genug am Tagesgeschäft, um krankenhausinternes Expertenwissen einzufangen und zu beurteilen. Obwohl Managementteams infolgedessen als „Säulen der Organisation“ gesehen werden können, sind ihre Aufgaben als „Brückenbauer“ doch äußerst schwer zu erfüllen. Von den jeweiligen Funktionsträgern wird viel erwartet, da strategische Entscheidungen der oberen Leitung zwar perfekt an die ökonomische Realität des Gesundheitsmarktes angepasst sind, aber der Realität und den Bedürfnissen der Krankenversorgung nicht unmittelbar gerecht werden. Hier braucht es kreative Übersetzungsleistungen, um Strategien feinzujustieren und praktisch umzusetzen.

\section{Raus aus der Sandwichposition,} rein in die Brückenbauerfunktion

Die mittlere Führungsebene steht im ständigen Kontakt mit den Mitarbeitern und ist Dreh- und Angelpunkt, Stabilität und Mitarbeiterbindung in der Organisation zu erreichen. Der „Engagement Index Deutschland 2011“ zeigt eindrucksvoll, wie wichtig eine hohe emotionale Bindung der Mitarbeiter an den Arbeitgeber ist (Abb. 1). Diese Mitarbeiter sind engagiert, loyal und produktiv, haben wenige Fehltage und eine geringe Fluktuation. Leider sind das nur 14\% der Befragten. Erschreckend ist v. a. auch die umgekehrte Erkenntnis: Mitarbeiter mit geringer emotionaler Bindung an den Arbeitgeber sind „unengagiert“, leisten Dienst nach Vorschrift und haben vergleichsweise eine höhere Anzahl an Fehltagen sowie eine höhere Fluktuationsrate. Erschreckend ist, wie hoch der Anteil der Mitarbeiter ist, die in diese Kategorie fallen (63\%). Hier ruht enormes ungenutztes Potenzial, das zu entfalten eines der Hauptaufgaben guter Mitarbeiterführung ist. Die 23\% Mitarbeiter ohne emotionale Bindung sind sogar „aktiv unengagiert“, d.h. sie zeigen unerwünschtes Verhalten, welches zu Lasten der Leistungs- und Wettbewerbsfähigkeit des Unternehmens gehen kann [5]. Anhand der Gallup-Studie wird deutlich, wie wichtig die Zufriedenheit der Mitarbei-

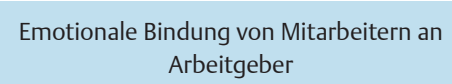

100

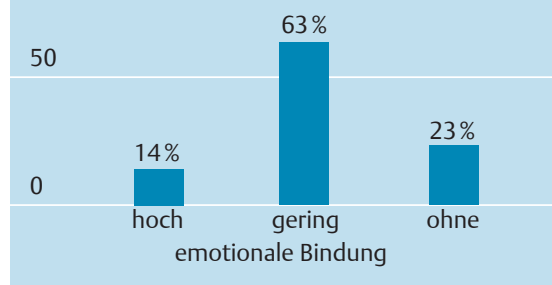

Abb. 1 Gallup-Studie - Engagement Index Deutschland 2011 [5].

ter mit der Organisation, mit dem Team und auch mit den Führungskräften des Krankenhauses für die Motivation und das Engagement der Mitarbeiter sind. Zufriedene Mitarbeiter mit einer hohen emotionalen Bindung an den Arbeitsplatz sind zudem unter dem Gesichtspunkt des Empfehlungsmarketings wichtig. So empfehlen 81\% der Mitarbeiter mit einer hohen emotionalen Bindung ihre Firma als einen hervorragenden Arbeitsplatz Freunden und Familienangehörigen, wohingegen dies nur 7\% der Arbeitnehmer ohne emotionale Bindung tun.

Mitarbeiterzufriedenheit sollte so insbesondere auch im Hinblick auf den gravierenden Fachkräftemangel ein ausschlaggebender Punkt zur Mitarbeitergewinnung und -bindung sein. Wenn die Gallup-Studie darauf hinweist, dass die Mitarbeiter eine offene und wertschätzende Kommunikation sowie ein ehrliches Interesse an ihren zentralen Bedürfnisse und Erwartungen vermissen, so zeigt gerade das doch den Ansatzpunkt besserer Mitarbeiterführung [5]. Gute Führung und gutes Management gehen nicht von alleine - dazu braucht es v.a. eine gewisse zeitliche Priorität.

\section{Teamerfolg durch mehr Augen- höhe mit den Mitarbeitern}

Die Mitarbeiterzufriedenheit wird stark von klaren, Sicherheit gebenden Verantwortlichkeiten und Verbindlichkeiten gelenkt. Die Effektivität und Effizienz der Prozesse auf Station ist abhängig davon, inwieweit das Managementteam aus Arzt und Pflege funktioniert und ob es gelingt, gemeinsam Verantwortung für die Station und für die Einbindung der Mitarbeiter zu übernehmen. Eine gute Organisation der Patientenversorgung, welche durch strukturierte und abgestimmte Stationsabläufe sowie klare Führungsstrukturen ermöglicht wird, ist am Ende ausschlaggebend für die Zufriedenheit der Mitarbeiter und der Patienten und damit für den nachhaltigen Erfolg der Organisation.

\section{Literatur}

1 Butler T, Waldroop J. Wie Unternehmen ihre Besten Leute an sich binden. Harvard Business Manager 2012; 2: 99-107

2 Dietrich M, Jacobs P, Eberts E. Entwicklung einer berufsgruppenübergreifenden Verantwortungskultur. Ein Beispiel am Klinikum der Universität München (KUM). Klinikarzt 2011; 2: 62-64

3 François-Kettner H, Schär W. Ausgewählte Führungsinstrumente an Beispielen der Pflegeprofession. Bundesverband Pflegemanagement, Kma-online, 24.01.2012. http:// www.kma-online.de/nachrichten/ id_26378_view.html (abgerufen am 10.04.2012)

4 François-Kettner H, Stern S, Eberts E, Kirsch S, Ruhl S. Stationskonzept „Pro Patient” ein Pilotprojekt der Charité und des ZeQ. Das Krankenhaus 2009; 8: 743-746

5 Gallup Studie „Engagement Index 2011“ eu.gallup.com/file/Berlin/153299/Gallup_ EEI_2011_Pr\%C3\%A4sentation_Pressekonferenz_Download.pdf (abgerufen am 18.04.2012)

6 Knoblauch J, Kurz J. Die besten Mitarbeiter finden und halten. Die ABC-Strategie nutzen. Frankfurt/New York: Campus Verlag, 2011

7 Krüttgen N, Ruhl S. Stationskonzepte. Neuverteilung der Aufgaben- und Kompetenzprofile. Klinikarzt 2012; 2: 69-71

Korrespondenz

Dr. Elke Eberts

Ruhl Consulting AG

Harrlachweg 1

68163 Mannheim

Tel.: 0621/ 3288649-0

E-Mail: elke.eberts@ruhl-consulting.de

Alexa Schmall

Ruhl Consulting AG

Harrlachweg 1

68163 Mannheim

Tel.: 0621/ 3288649-0

E-Mail: alexa.schmall@ruhl-consulting.de

Hedwig Francois-Kettner

Pflegedirektorin

Charité - Universitätsmedizin Berlin

Charitéplatz 1

10117 Berlin

Tel.: 030/ 450 - 577021

E-Mail: francois-kettner@charite.de 